\title{
Influence of SiC Grain Boundary Character on Fission Product Transport in Irradiated TRISO Fuel
}

\author{
T. M. Lillo, ${ }^{\mathrm{a}}$ I. J. van Rooyen ${ }^{\mathrm{b}}$ \\ ${ }^{a}$ Materials Science and Engineering Department, Idaho National Laboratory, \\ Idaho Falls, ID 83415-2211, USA \\ ${ }^{b}$ Fuel Performance and Design Department, Idaho National Laboratory, \\ Idaho Falls, ID 83415-6188, USA
}

\begin{abstract}
In this study, the fission product precipitates at silicon carbide grain boundaries from an irradiated TRISO particle were identified and correlated with the associated grain boundary characteristics. Precession electron diffraction in the transmission electron microscope provided the crystallographic information needed to identify grain boundary misorientation and boundary type (i.e., low angle, random high angle or coincident site lattice (CSL)related). The silicon carbide layer was found to be composed mainly of twin boundaries and small fractions of random high angle and low angle grain boundaries. Most fission products were found at random, high-angle grain boundaries, with small fractions at low-angle and CSL-related grain boundaries. Palladium (Pd) was found at all types of grain boundaries while Pd-uranium and Pd-silver precipitates were only associated with CSL-related and random, high-angle grain boundaries. Precipitates containing only Ag were found only at random, high-angle grain boundaries, but not at low angle or CSL-related grain boundaries.
\end{abstract}

Key words: TRISO, SiC, grain boundary character, fission product transport

* Corresponding author. Tel +1 2085269746

E-mail address: thomas.lillo@inl.gov

\section{INTRODUCTION}

The silicon carbide (SiC) layer of tristructural isotropically (TRISO) coated nuclear fuel particles has been carefully engineered to act as the primary containment for fission products in high-temperature gas reactors; however, significant release of certain fission product elements through seemingly intact fuel particles has been reported in a number of irradiation experiments [1-5].The body of research over the last 40 years strongly suggests that grain boundaries are the primary pathway for fission product migration through the $\mathrm{SiC}$ layer. This theory is supported by results of recent electron microscopy examinations of neutronirradiated silicon carbide (SiC) from TRISO-coated particles from the US Department of Energy's Advanced Gas Reactor (AGR)-1 experiment, which found strong evidence of fission products at SiC grain boundaries [6-8]. Meanwhile, bulk diffusion of fission product elements, especially Ag, in $\mathrm{SiC}$ has been shown to be negligible [e.g. 9-14] and fission product release via macroscopic defects, such as microcracks [15] and nanotubes [16], in neutron-irradiated TRISO fuel during in-pile tests has not been substantiated.

If transport by grain boundaries is the dominant mechanism for fission product movement through the $\mathrm{SiC}$ layer, it follows that grain boundaries (and individual grain boundary characteristics $[17,18]$ ) play an important role in the release of fission products in TRISO fuel. Rabone et al. [19] have shown through computational simulation that SiC grain boundary characteristics can significantly affect the diffusion of fission product elements and that those effects are fission product element-specific. Furthermore, previous analyses of an irradiated TRISO particle showed that not all grain boundaries in the SiC layer contain fission products and those precipitates are either individual fission product elements or specific 
combinations [7]. These observations suggest that an understanding of the relationship between grain boundary character and fission product migration will advance the understanding of fission product release from TRISO coated fuel particles. To this end, the $\mathrm{SiC}$ layer from an irradiated TRISO coated nuclear fuel particle was characterized for grain boundary characteristics and fission product location/composition and the results of these analyses were correlated. A method using precession electron diffraction (PED) in the transmission electron microscope (TEM) on the SiC layer of an unirradiated TRISO fuel particle has been demonstrated to quickly and efficiently provide the crystallographic information needed to identify grain boundary misorientation, grain boundary type (low or high angle) and whether the boundary is coincident site lattice (CSL)-related [20]. In the work described here, this method is applied to TEM samples prepared from the SiC layer of an irradiated TRISO coated nuclear fuel particle to characterize the influence of grain boundary parameters on fission product migration.

\section{EXPERIMENTAL}

The samples from the irradiated TRISO fuel particle studied in Reference [7] were used in this study and detailed information on their origin and fabrication can be found in the referenced document. However, to summarize, the TEM samples in this study originated from Particle 35 from Compact 6-3-2 in the AGR-1 irradiation experiment in the Advanced Test Reactor at Idaho National Laboratory and was determined to retain a relatively high fraction of its expected Ag-110m inventory [1]. The TRISO particles in this compact were fabricated at Oak Ridge National Laboratory using baseline conditions, which for the SiC layer consisted of $\mathrm{H}_{2}+$ MTS coating gas at a coating gas fraction of about 0.012 and $1500^{\circ} \mathrm{C}$ for 140 minutes [21]. This produced an average SiC layer thickness of $35.3 \mu \mathrm{m}$ and an average density of $3.20 \mathrm{~g} / \mathrm{cm}^{3}$ [21]. The nominal irradiation conditions of this particle are shown in Table 1 . However, it must be kept in mind that this particle was part of a compact typically containing over 4,100 TRISO particles. Therefore, the actual irradiation conditions experienced by this one specific particle may have been significantly different due to specific location in the compact, which could not be documented.

Table 1. Irradiation parameters for TRISO particle.

\begin{tabular}{lc}
\hline \multicolumn{1}{c}{ Parameter } & Value \\
\hline \% FIMA average burnup & 11.3 \\
Time-average, volume-average temperature, ${ }^{\circ} \mathrm{C}$ & 1070 \\
Time-average, maximum temperature, ${ }^{\circ} \mathrm{C}$ & 1144 \\
Approximate time-at-temperature, days & 620.2 \\
Approximate fast fluence, $\mathrm{n} / \mathrm{cm}^{2}$ & $2.38 \times 10^{21}$ \\
Fuel type & Baseline \\
\hline
\end{tabular}

Three TEM samples were prepared by standard focused ion beam methods and were taken from areas at the inner pyrolytic carbon (IPyC)/SiC interface, mid-thickness of the SiC layer, and at the SiC/outer pyrolytic carbon (OPyC) interface to obtain at least qualitative information as a function of distance through the thickness of the SiC layer. Subsequently, these samples will be referred to as inner, center, and outer, respectively. In general, the center sample overlaps both the inner and outer samples to some degree (see Figure 1).

Fission products and transuranic elements in each sample were located using z-contrast imaging in scanning transmission electron microscopy and using a high-angle annular darkfield detector. The composition of intergranular precipitates were then analyzed using a fine electron probe size (i.e., about $5 \mathrm{~nm}$ ) (Tecnai TF30-FEG STwin, operating at $300 \mathrm{kV}$, at 
the Center for Advanced Energy Studies) and energy dispersive spectroscopy. As in a previous study [7], the limits of detection for fission product elements were taken as $0.2 \mathrm{wt} \%$. (The beam size was on the order of the precipitate size and precipitate compositions were considered to be qualitative.)

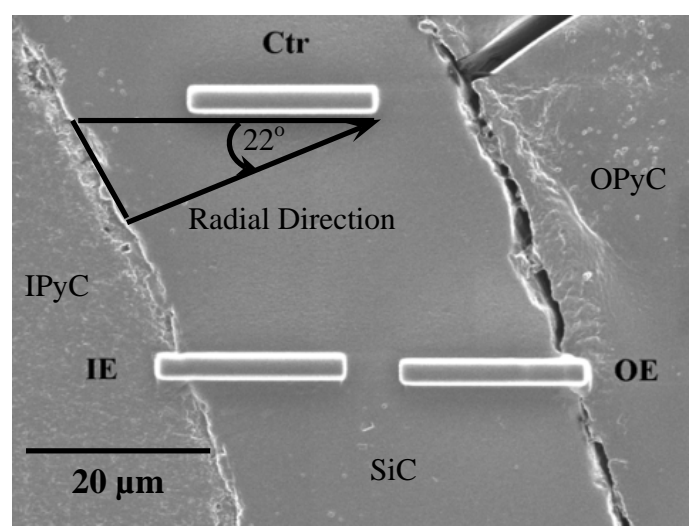

Figure 1. Focused ion beam-prepared TEM samples were taken from the locations indicated in this photo: inner (IE), center (Ctr), and outer (OE). The center sample significantly overlaps the inner and outer samples.

Following fission product location and composition identification, a scan of the area containing the fission product precipitate using PED was performed to analyze the crystallographic orientation of the grains in the vicinity of the precipitate. The ASTAR system (NanoMegas, Inc.) was used to generate and collect the PED information. An electron probe size of about $5 \mathrm{~nm}$, in conjunction with a 10-nm step size, was used in data collection. The crystallographic information was exported and analyzed using EDAX OIM v7.1.0 software. Two data cleanup routines were applied to all data and consisted of the following:

1. Grain dilation: grain tolerance angle is 2 degrees and minimum grain size is 5 pixels

2. Neighbor orientation correlation: grain tolerance is 2 degrees and minimum confidence index is 0 with a cleanup level of 1 . (For a cleanup level of 1 , all but one of the nearest neighbor data points must be of the like orientation before the datapoint in question will be changed to the like orientation of the nearest neighbors. For this work the data points were collected in a hexagonal pattern and, thus, 5 of 6 nearest neighbors were required to have the same orientation before the orientation of the data point was changed to match the 5 nearest neighbors.)

The cleanup routines above are highly restrictive to changing the orientation of any given data point and, generally, less than $3 \%$ of the data points were affected by the cleanup routines. This well below the limit of 10\% allowed in Section 12.2 of ASTM Standard E2627 which provides guidance on data processing for the use of EBSD in the determination of grain size [22]. Grain boundaries were defined at a misorientation equal to or greater than 2 degrees. The range of CSL-related grain boundaries was defined to include $\Sigma 3$ through $\Sigma 29$.

\section{RESULTS}

\subsection{General Microstructure}

When analyzing grain orientations in the vicinity of specific fission product precipitates, an area of approximately $2 \mu \mathrm{m} \times 2 \mu \mathrm{m}$ was analyzed. This area also contained a large number of grain boundaries that did not exhibit fission product precipitates. All grain boundaries in each 
of the areas analyzed in the inner, center, and outer samples were compiled to compare the general grain boundary distribution across the thickness of the SiC layer. Typically, the individual areas of analysis were not large enough to capture the larger grains; therefore, an accurate grain size determination through the SiC layer was not possible, meaning the characterization efforts were limited to grain boundary orientation and character distributions.

Figure 2 shows a representative example of the microstructure in one area of analysis on each of the three samples. The TEM images are shown along the top row while the orientation map of the region outlined in white dots is shown in the bottom row. In each orientation map, in the bottom row, the high-angle boundaries are outlined in black while the low-angle grain boundaries are shown in white. The images in Figure 2 show that most of the grain boundaries are high angle with relatively few low-angle grain boundaries present. Qualitatively, the grain size in the inner area (Figure 2a and d) appears to be smaller than in the areas from the center and outer samples, as expected from $\mathrm{SiC}$ grown by chemical vapor deposition (CVD) methods [23-25] and in agreement with the variation in grain size of SiC in unirradiated, baseline TRISO fuel particles as reported by Kirchhofer [26].

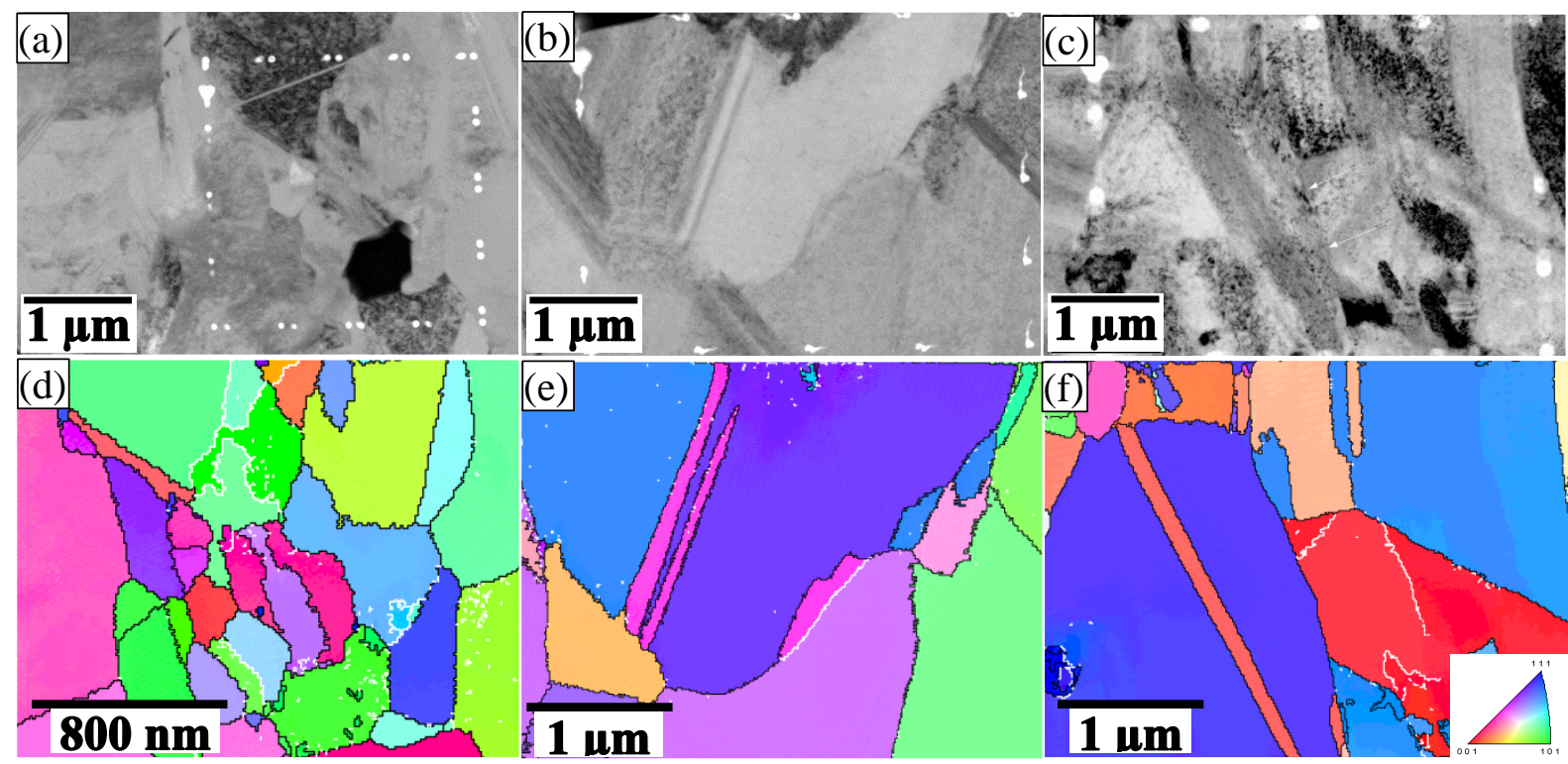

Figure 2. Examples of SiC grain structure, TEM image top row, and the associated orientation map (inverse pole figure) of the area outline in white dots, bottom row, taken from one area in (a and d) the inner, (b and e) center, and (c and f) outer samples. High-angle grain boundaries are in black, while lowangle boundaries are in white.

\subsection{General Grain Boundary Distributions}

The grain orientation information provided by PED was used to determine the attributes of all grain boundaries in the analyzed areas of all three samples. Data from all analyzed areas on a given sample were compiled to generate various frequency distributions for each sample.

Misorientation Angle Distribution: Figure 3 shows the grain boundary misorientation angle frequency distribution found in the inner, center, and outer samples. The distribution corresponding to the inner sample was generated from misorientation of 445 grain boundaries, while the center distribution was generated using 315 grain boundaries and the outer using 168 grain boundaries. The error bars represent the standard error in each misorientation angle category. It should be kept in mind that the center sample contains significant overlap with both the inner and outer samples (see Figure 1). 
The distribution in Fig. 3a is based on the number fraction of grain boundaries (i.e. the number of grain boundaries with a given misorientation angle divided by the total number of grain boundaries in the analyzed area) while the distribution in Fig. 3b is based on the length fraction of grain boundaries (i.e. the length of grain boundary with a given misorientation divided by the total length of grain boundaries in the analyzed area). The number-based distribution and the length-based distribution exhibit the same trends but slightly different fractions for each misorientation, especially the low angle grain boundaries (misorientation angle $\leq 15^{\circ}$ ). The data in the two distributions in Fig. 3 indicate that there are relatively few low angle grain boundaries but they are generally longer in length and thus, low angle grain boundaries exhibit a relatively low fraction in the number-based distribution of Fig. 3a and a significant higher fraction in the length-based distribution of Fig. $3 \mathrm{~b}$.

In general, the three distributions are quite similar. Low-angle grain boundaries make up approximately $10 \%$ of the analyzed boundaries in each sample. Twin boundaries, with a 60degree misorientation angle, represent a majority of the grain boundaries in the SiC layer. A smaller peak in the distribution occurs around 40 degrees. The only difference among the three distributions lies in the frequency of the twin boundaries. Significantly fewer twins are in the inner sample, where growth of the SiC layer began compared to the outer sample, where competitive growth during CVD has downselected grains with favorably oriented fast growth directions.
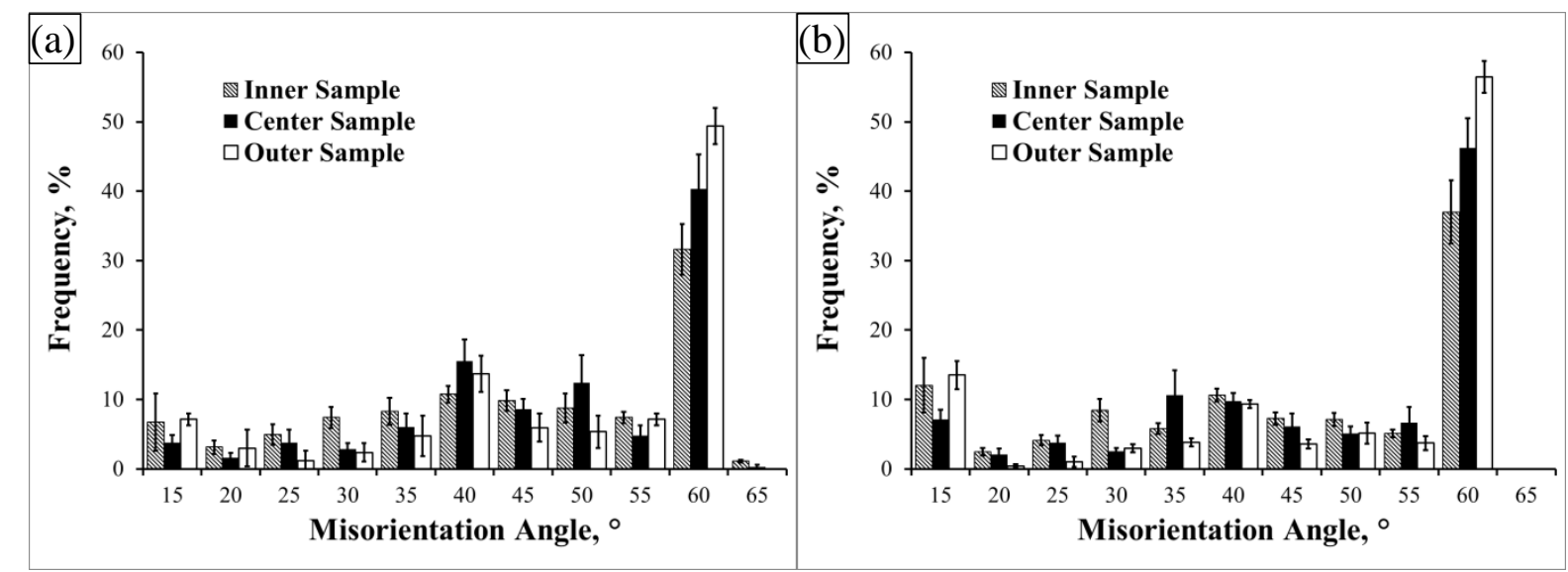

Figure 3. Plots of the grain boundary misorientation angle distribution for the inner, center, and outer samples. The number of grain boundaries was used as the basis in (a) while the grain boundary length was used as the basis in (b).

Distribution by Grain Boundary Type: The grain boundary information may be further analyzed to determine the relative amounts of the different types of grain boundaries - low angle, random high angle, and CSL-related grain boundaries (only $\Sigma 3$ through $\Sigma 29$ are considered). The distribution found in each sample is summarized in Figure 4, in the form of pie charts. Again, number fraction has been used as the basis in Fig. 4a,c,e while grain boundary length fraction has been used as the basis in Fig. 4b,d,f. The fraction of CSL-related grain boundaries increases from the inner sample to the outer sample, while the fraction of random high-angle grain boundaries diminishes steadily from the inner to the outer sample. The fraction of low-angle grain boundaries is relatively constant across the thickness of the SiC layer.

The trends in the grain boundary character distribution found here follow those obtained by Kirchhofer [26] from SEM EBSD analysis of the SiC layer in an unirradiated, TRISO fuel particle fabricated under baseline conditions. As in that work, CSL-related boundaries were 
found to be most prevalent here, averaging 59\% (grain boundary length basis) across the entire SiC layer, and random, high angle grain boundaries next most common at an average of $\sim 30 \%$. Low angle grain boundaries were least common at an average of $\sim 11 \%$. Kirchhofer [26], however, reports a considerably higher fraction of low angle grain boundaries at 24\% (grain boundary length basis) and values of $40 \%$ and 36\% for CSL-related and random, high angle grain boundaries, respectively, although statistical variation for these fractions is not given. The main difference between the two studies is unirradiated $\mathrm{SiC}$ for the Kirchhofer study versus high temperature $\left(\sim 1100^{\circ} \mathrm{C}, \sim 14,900\right.$ hours), irradiated $\mathrm{SiC}$ for this study. Additionally, the data collection step size in the Kirchhofer study was $\sim 5$ times larger than in this study. The differences in the grain boundary character distributions may be attributable to either experimental difference. While the irradiation temperature represents approximately less than half the melting point of $\mathrm{SiC}$, enhanced diffusion due to irradiation-induced defects, in combination with a long exposure duration, may allow significant rearrangement of lattice dislocations and alter the relative fraction of low angle grain boundaries compared to unirradiated SiC. Conversely, if lattice dislocations, potentially arising from the CVD fabrication of the SiC layer, are distributed relatively uniformly throughout grain interiors, a larger data acquisition step size could result in an apparent low angle grain boundary, if enough dislocations exist between adjacent data points to exceed the minimum misorientation angle defined in the EBSD analysis software ( $2^{\circ}$ in both studies). Additionally, each study is based on the analysis of a single TRISO particle and the particle-to-particle variation in microstructure is not known at this time. Therefore, grain boundary character distribution studies on additional particles in the as-fabricated and irradiated conditions, using the same data collection technique and step size, will be required to determine the cause of the differences discussed here, if the differences are, indeed, statistically significant.
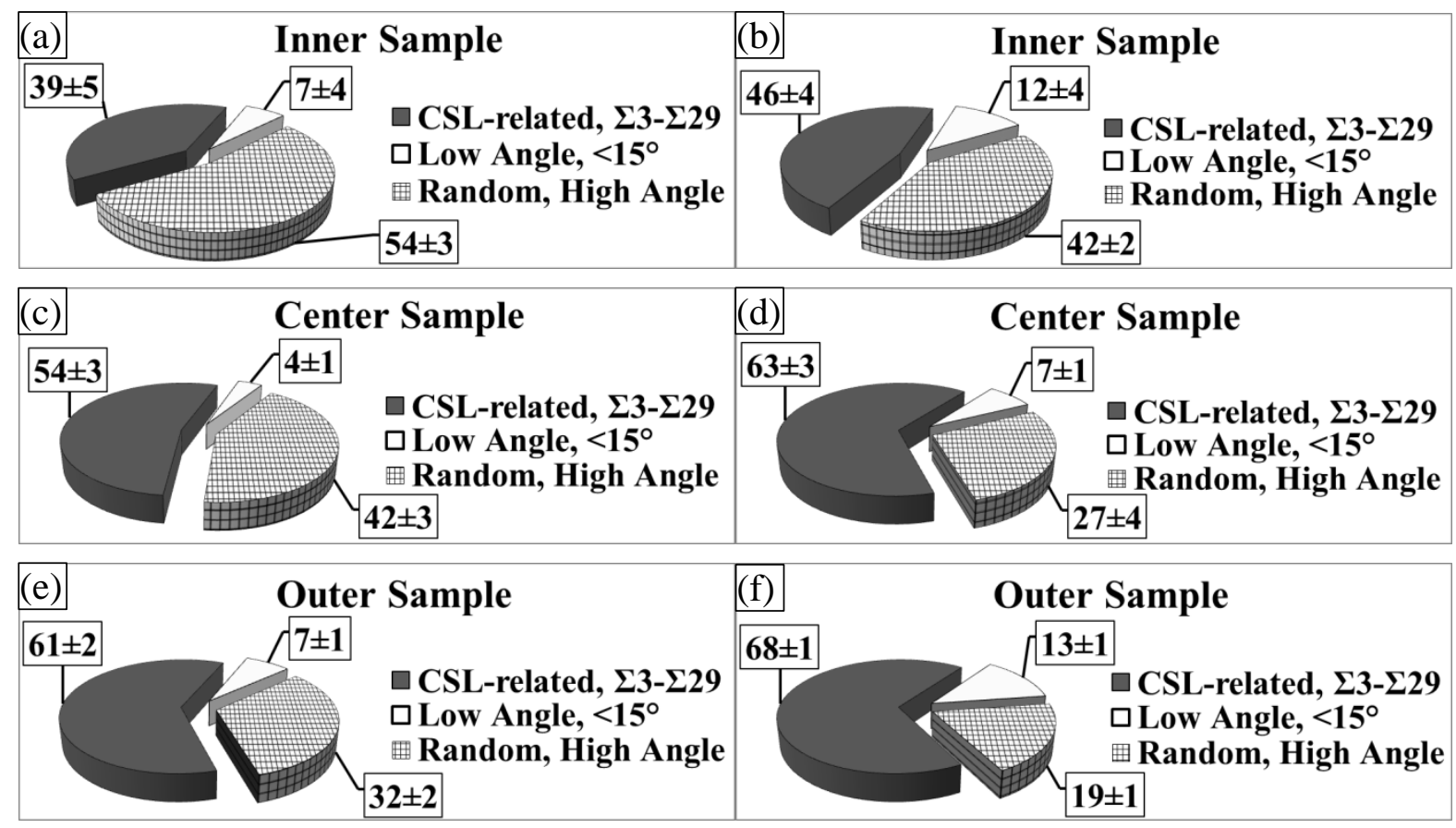

Figure 4. Distribution, as a percentage, of CSL-related $(\Sigma)$, low-angle and random, high-angle grain boundaries for $(a, b)$ the inner, $(c, d)$ center and $(e, f)$ outer samples, using the number of grain boundaries as the basis $(a, c, e)$ and $(b, d, f)$ the same distributions using the grain boundary length as the basis.

Distribution of CSL-Related Grain Boundaries: As mentioned, the twin boundary fraction appears to dominate in the misorientation angle distribution in Figure 3. This is confirmed in the distribution by CSL boundary type (indicated by the $\Sigma$ value) in Figure 5 . The $\Sigma 3$ boundary type, which corresponds to the twin boundary, is most prevalent of the CSL-related 
grain boundaries in all three samples. The higher order twins (i.e. $\Sigma 9$ and $\Sigma 27$ ) make up the majority of the remaining CSL-related grain boundaries. The fraction of grain boundaries that are CSL-related is increasing from the inner sample to the outer sample (Figure 4) and it appears the increase in the fraction of twin-related boundaries, $\Sigma 3^{\mathrm{n}}$, is mainly responsible for the overall increase in the CSL boundary fraction from inner to outer areas (the fraction of non-twin related CSL boundaries remains relatively constant across the thickness of the SiC layer).

The boundary length fractions of $\Sigma 3^{\mathrm{n}}$ grain boundaries in these irradiated SiC samples are consistent with those reported by Kirchhofer [26] in unirradiated SiC made under the same conditions. The $\Sigma 3$ boundary length fraction found here - averaging $\sim 4 \% \%$ (grain boundary length basis) across the entire SiC layer - is slightly higher than that reported for unirradiated $\mathrm{SiC}, \sim 29 \%$ (grain boundary length basis) [26]. The length fractions of the $\Sigma 9$ and $\Sigma 27$ boundaries in this work, at $\sim 4 \%$ and $1 \%$, respectively, are identical to those reported by Kirchhofer [26]. The reason for the difference in the $\Sigma 3$ boundary fractions between the two studies is not clear but could be statistical variation in the analyses or particle-to-particle variation.
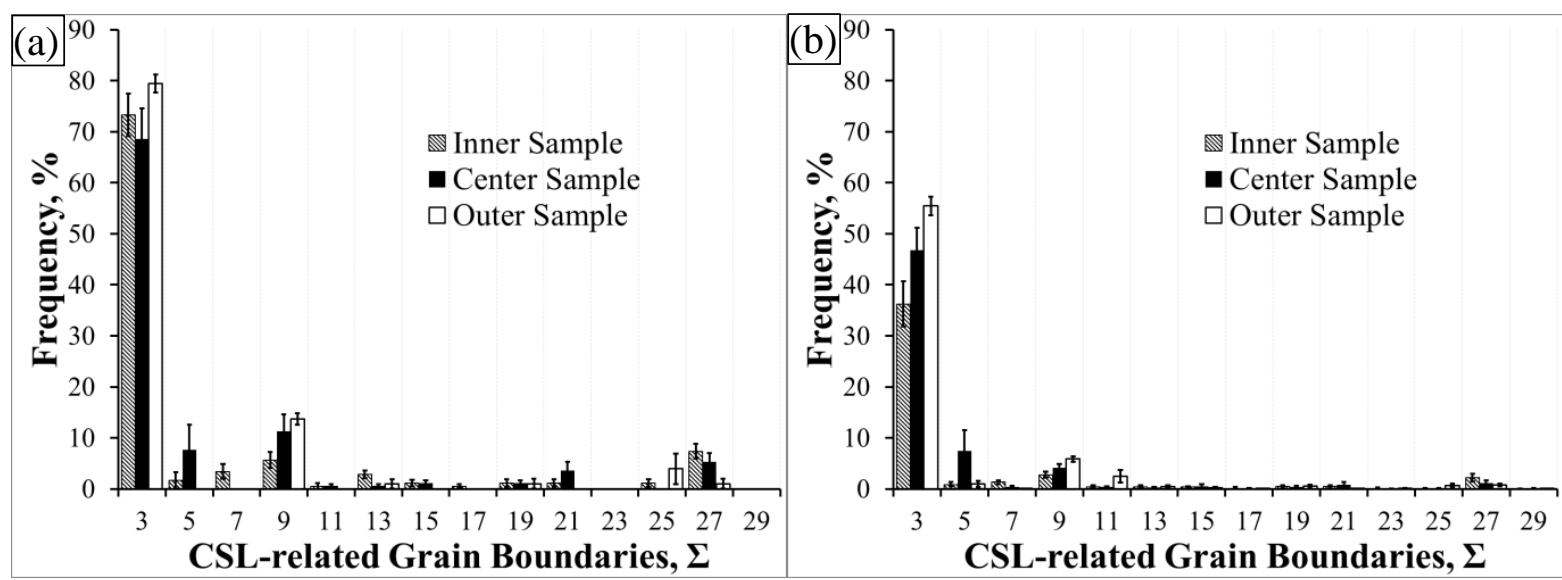

Figure 5. Distribution of the CSL-related grain boundaries by $\Sigma$ value, (a) number fraction and (b) length fraction.

\subsection{Grain Boundary Data for Grain Boundaries Containing Fission Products}

\subsubsection{Example of One Area with Fission Products}

Figure 6 shows one area with fission product precipitates that were analyzed for composition and the associated grain boundary information, mainly as an example of the type of information that was obtained from analysis areas. In this particular area, seven grain boundary segments exhibited fission product precipitates. Even though all segments were connected, the fission products present in each segment were not necessarily the same (Table 2). Most segments contain Pd with the exception of boundary segments 2 and 3, which contain only Ag. In this example, it appears that the grain boundary parameters, which determine grain boundary energy and grain boundary atomic structure, affect which fission products may be present on a particular grain boundary segment. This is somewhat surprising since boundary segments 1, 2, 3, and 4 in Table 2 are all considered to be random, high-angle grain boundaries - all are expected to be of relatively high energy and with no expected specific repeating atomic structure as they are random grain boundaries - and yet they exhibit elevated levels of different fission product elements. They must possess unique characteristics, energy or atomic structures, which allow preferential segregation of certain 
fission product elements to them. Since boundaries 1-4 are linearly connected, one would expect to find the fission products on all four grain boundaries to be the same with approximately the same concentration if all random, high angle boundaries were equivalent, which is clearly not the situation in this example. At the very least, the specific boundary parameters strongly influence the diffusion of the various fission products.
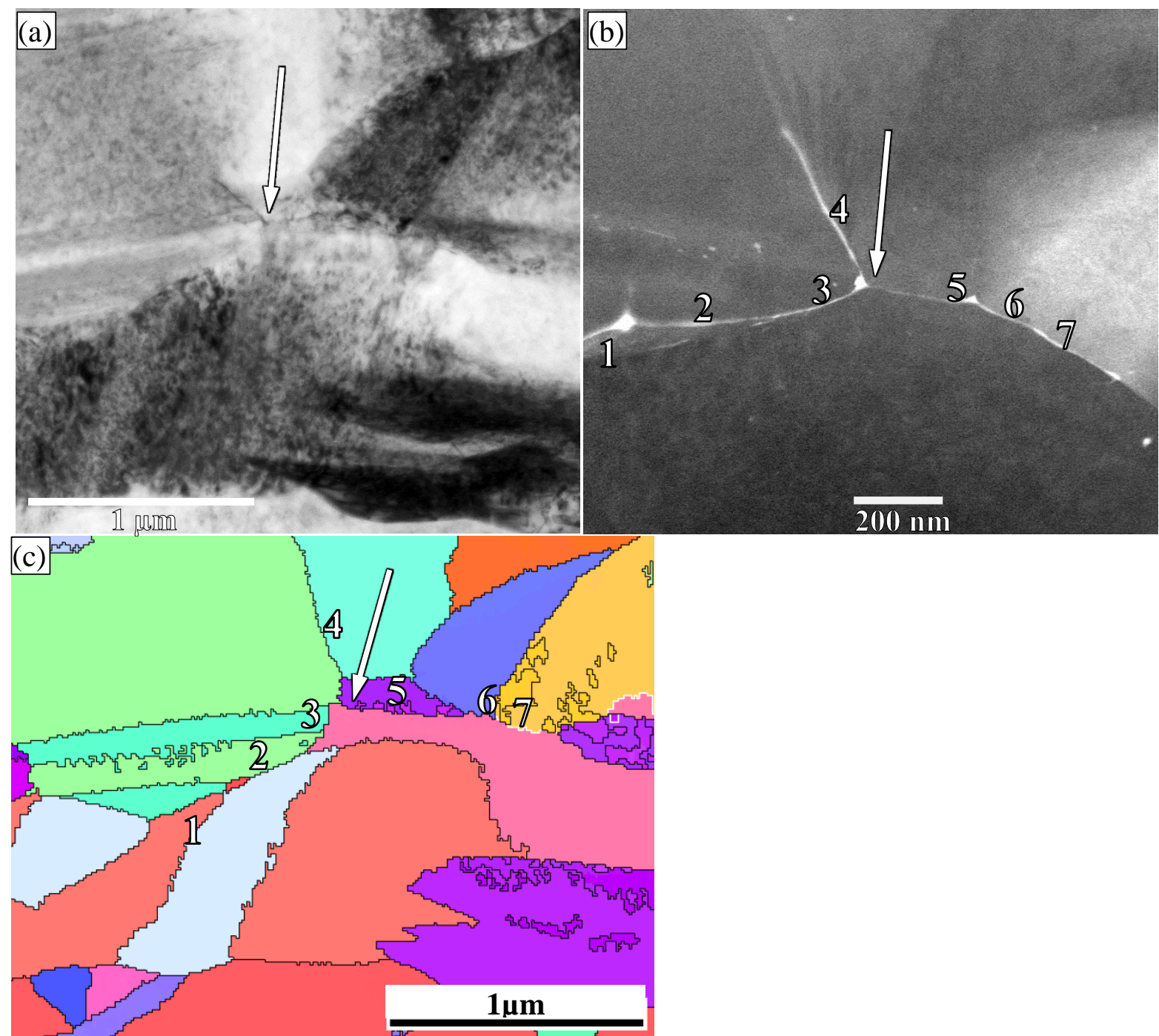

Figure 6. Example area showing grain boundary precipitates that were analyzed for composition and grain boundary character. (a) TEM image, (b) scanning transmission electron microscopy image using the high-angle annular darkfield detector and (c) the corresponding orientation image. The arrow in each image denotes the same reference point.

Table 2. Summary of fission product precipitate information and associated grain boundary information.

\begin{tabular}{|c|c|c|c|c|c|c|}
\hline Grain Boundary & Angle & Axis & CSL designation & $\mathrm{Pd}$ & $\mathrm{Ag}$ & $\mathrm{U}$ \\
\hline 1 & 33.6 & $20 \quad 13-13$ & - & $\bullet$ & & $\bullet$ \\
\hline 2 & 45.7 & 51315 & - & & - & \\
\hline 3 & 54.2 & $18 \quad 2 \quad-15$ & - & & $\bullet$ & \\
\hline 4 & 43.6 & $\begin{array}{lll}4 & 16 & -19\end{array}$ & - & - & & \\
\hline 5 & 38.7 & $\begin{array}{lll}1 & 0 & 1\end{array}$ & 9 & $\bullet$ & & \\
\hline 6 & 56.8 & $20-123$ & - & $\bullet$ & & \\
\hline 7 & 13.8 & $\begin{array}{lll}-7 & 23 & -18\end{array}$ & - & $\bullet$ & & \\
\hline
\end{tabular}




\subsubsection{Summary of Precipitate Composition and Associated Grain Boundary Information}

Analyses similar to the example in the previous section were carried out on the various areas of each sample taken from the SiC layer. The following summarizes the combined results of the areas analyzed in each sample.

Area Nearest IPyC (Inner Sample): Grain boundary precipitates were most prevalent in the $\mathrm{SiC}$ layer near the IPyC layer (Figure 7), which is as expected because this location is nearest the source of the fission products, i.e. the central fuel kernel. Sixty-three boundaries were analyzed that exhibited fission product precipitates in this sample. The majority of the fission product precipitates contain only Pd (about 57\%) or Pd in conjunction with U (about 22\%), Table 3. Fission product precipitates exhibiting only Ag were present in significant quantities at about $13 \%$, as were fission product precipitates that contained both Pd and $\mathrm{Ag}$ at about $8 \%$. Table 3 also shows that fission product precipitates in the inner sample had the highest probability of occurring at random, high-angle type grain boundaries, regardless of the fission product element(s) present. Precipitates consisting of only Ag were found only on random, high-angle type grain boundaries, whereas Pd only, $\mathrm{Pd}+\mathrm{Ag}$, and $\mathrm{Pd}+\mathrm{U}$ were found occasionally on CSL-related grain boundaries, but were predominantly found on random, high-angle grain boundaries. The fission product precipitate on the single low-angle grain boundary contained Pd exclusively.

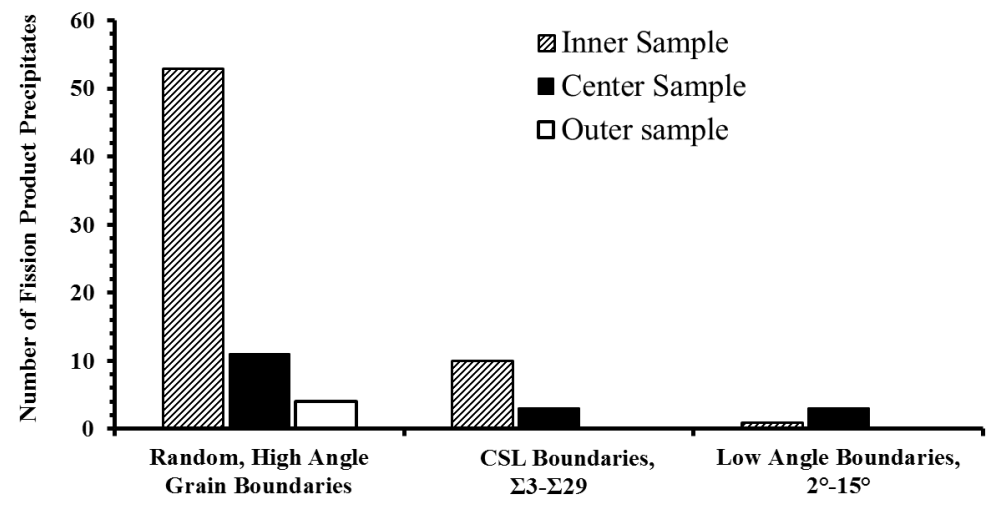

Figure 7. Summary of the grain boundary types associated with fission products.

Table 3. Summary of the distribution of fission product elements.

\begin{tabular}{|c|c|c|c|c|c|c|}
\hline & Pd only & Ag only & U only & Pd \& Ag & Pd \& U & Ag \& U \\
\hline \multicolumn{7}{|l|}{ Inner Sample } \\
\hline Number of grain boundaries & 37 & 8 & 0 & 5 & 14 & 0 \\
\hline Fraction of all boundaries with fission products, \% & 57.8 & 12.5 & 0.0 & 7.8 & 21.9 & 0.0 \\
\hline Low angle, $\%$ & 2.8 & 0.0 & - & 0.0 & 0.0 & - \\
\hline Random, high angle, \% & 80.6 & 100.0 & - & 80.0 & 85.7 & - \\
\hline CSL, \% & 19.4 & 0.0 & - & 20.0 & 14.3 & - \\
\hline \multicolumn{7}{|l|}{ Center Sample } \\
\hline Number of grain boundaries & 12 & 4 & 0 & 0 & 1 & 0 \\
\hline Fraction of all boundaries with fission products, \% & 70.6 & 23.5 & 0.0 & 0.0 & 5.9 & 0.0 \\
\hline Low angle, $\%$ & 25.0 & 0.0 & - & - & 0.0 & - \\
\hline Random, high angle, \% & 50.0 & 100.0 & - & - & 100.0 & - \\
\hline CSL, \% & 25.0 & 0.0 & - & - & 0.0 & - \\
\hline \multicolumn{7}{|l|}{ Outer Sample } \\
\hline Number of grain boundaries & 4 & 0 & 0 & 0 & 0 & 0 \\
\hline Fraction of all boundaries with fission products, \% & 100.0 & 0.0 & 0.0 & 0.0 & 0.0 & 0.0 \\
\hline Low angle, \% & 0.0 & - & - & - & - & - \\
\hline Random, high angle, \% & 100.0 & _ & _ & - & _ & _ \\
\hline CSL, \% & 0.0 & - & - & - & - & - \\
\hline
\end{tabular}


Middle of the SiC Layer (Center Sample): The number of precipitates in the center sample was much lower compared to the inner sample. As a result, only 17 fission product precipitates and their associated grain boundary type were analyzed in this sample. However, the trends observed for the inner sample appear to be followed in the center sample (Table 3). Again, most of the fission product precipitates contain Pd and are associated with random, high-angle grain boundaries. However, smaller fractions of Pd-containing precipitates are associated with either CSL-related or low-angle grain boundaries. As in the inner sample, precipitates containing only Ag were found exclusively on random, high-angle grain boundaries and were never observed on either CSL-related or low-angle grain boundaries. In this sample, only one precipitate containing Pd in conjunction with U was found on a random, high-angle grain boundary. Observations in the inner sample found similar $\mathrm{Pd} / \mathrm{U}$-containing precipitates occur to a small degree on CSL-related grain boundaries. It is possible that $\mathrm{Pd} / \mathrm{U}$-containing grain boundary precipitates would be found on CSL-related grain boundaries in the middle of the $\mathrm{SiC}$ layer if analyses on additional precipitates in additional samples taken from the center of the SiC layer were performed. No Pd/Ag-containing precipitates were found in this sample.

Outer Edge: Only four fission product precipitates and their associated grain boundary information were analyzed in the outer sample. The number of fission product precipitates was very low, possibly due to the relatively large grain size in this part of the SiC layer, the relatively long diffusion distances involved and/or potentially low fission product concentrations that were insufficient to form a precipitate. In this sample, all fission product precipitates were found to contain Pd exclusively and only found on random, high-angle type grain boundaries (Table 3). Precipitates with other elements may be present in other outer areas of the $\mathrm{SiC}$ layer that were not analyzed in this study. However, an impractical number of TEM samples would be required to determine these occurrences with any reasonable certainty. The combination of increasing grain size, increasing diffusion distance, and changing grain boundary distribution (see Figure 4) are thought to contribute to the decreasing precipitate density in this region.

\section{DISCUSSION}

\subsection{Associations of Fission Products with Grain Boundary Types $-\Sigma$, Low Angle, High Angle}

Pd-containing precipitates (i.e., Pd only and Pd with $\mathrm{U}$ ) were the most common throughout the SiC layer (Table 3 and Figure 8). Ag-containing (i.e., Ag only and Pd with Ag) were the next most common precipitate type. This is expected based on the higher Pd generation rate compared to that of Ag during irradiation of the TRISO fuel particle [27]. The source of U most likely originated from the UCO fuel kernel either due to contamination during the CVD fabrication of the SiC layer [28] or as a result of reducing conditions in the kernel imposed by the adjacent carbon buffer and IPyC layers during irradiation that enabled $U$ to migrate into the $\mathrm{SiC}$ layer. However, U precipitates were not observed in examinations of the as-fabricated SiC layer of unirradiated TRISO fuel [20]. Therefore, it is likely that the U migrated into the $\mathrm{SiC}$ layer during irradiation. Because $\mathrm{U}$ precipitates are not found in the interior of the $\mathrm{SiC}$ grains, but is always found associated with Pd (Table 3) on grain boundaries, it is likely moving with the assistance of Pd. Both Pd and U form silicides and $U$ has considerable solubility in metallic Pd [29] so it is reasonable to assume significant interaction between Pd and $U$ under the irradiation conditions, with $U$ being in a solid solution with metallic Pd or a 
Pd-based compound [6]. Further understanding of the precipitation behavior will require the definitive identification of the precipitates as to whether they are metallic or silicides.

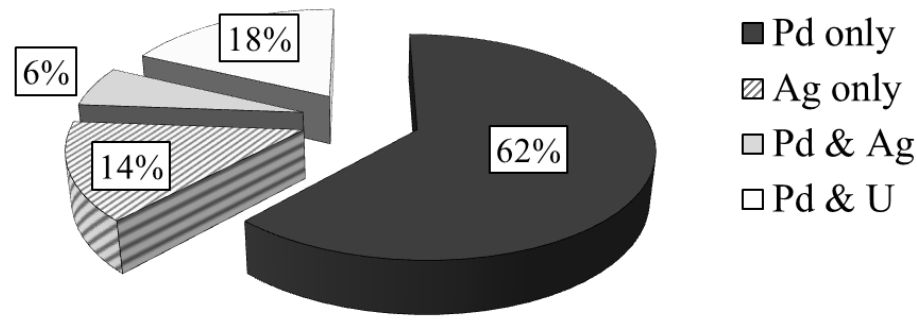

Figure 8. Composition distribution of the grain boundary precipitates.

The relatively low number of precipitates in this study prevents specific conclusions to be made concerning the effects of specific grain boundary misorientation parameters on the migration of different fission product elements through the SiC layer with any reasonable statistical certainty. However, it appears Pd more readily moves through the SiC grain boundary network than either Ag or U (Table 3), because very few Ag and U-containing precipitates were found beyond the region nearest the IPyC. This has been noted previously in a study by the authors [7] where only precipitate composition was assessed. In that study, Ag and $\mathrm{U}$ were found to have migrated only part way through the SiC layer during irradiation in the AGR-1 experiment, whereas Pd precipitates were found throughout the entire SiC layer. However, it is interesting to note that in the outer sample, Pd precipitates were only found on random, high-angle grain boundaries and not on low-angle or CSL-related grain boundaries. They did occur in small numbers on these types of grain boundaries in the inner and center samples (Table 3). One possible explanation is that, although Pd can migrate along these types of grain boundaries, the migration rate on low-angle and CSL-related grain boundaries is significantly lower than on random, high-angle grain boundaries. The $\mathrm{Pd}+\mathrm{U}$ and the $\mathrm{Pd}+\mathrm{Ag}$ precipitate distributions tend to also support this explanation. These precipitate types were present on CSL-related grain boundaries in the inner sample but not in the center sample. Table 3 shows that both Ag and U were present in the analyzed regions of the center sample on random, high-angle grain boundaries. If the migration rate along CSL-related grain boundaries were on the same order as the random boundaries, then these instances had a reasonable chance of being detected. However, it is likely that the migration rate along CSLrelated boundaries is dependent on the CSL value through grain boundary energy. Lower energy grain boundaries have been demonstrated to have lower diffusivity [30,31] and generally, CSL-related grain boundaries have lower energy than random, high angle grain boundaries, although the grain boundary plane can have a considerable influence on the grain boundary energy [32-34]. Table 4 summarizes the CSL-related boundaries with precipitates on them by fission product element. In the case of $\mathrm{Pd}+\mathrm{Ag}$ and $\mathrm{Pd}+\mathrm{U}$, the CSL-related boundaries exhibit high values of $\Sigma$ and are not found on the lower $\Sigma$ value grain boundaries, e.g. $\Sigma 3$, which tend to exhibit the lowest grain boundary energy. Palladium, with an atomic radius smaller than either Ag or $\mathrm{U}$, seems to be less affected by the $\Sigma$ value and even observed in one instance to reside on a twin boundary. This is not overly surprising because palladium precipitates have been found in the interior of the $\mathrm{SiC}$ grains [7], where the local atomic registry is perfect and the interaction energy to accommodate the mismatch of atomic radii between $\mathrm{Pd}, \mathrm{Si}$, and $\mathrm{C}$ would be expected to be higher than in a grain boundary which has associated excess free volume. 
Of course, the other explanation for a lack of Pd precipitates on low angle and CSL-related grain boundaries in the outer sample is that simply not enough precipitates were analyzed in the outer sample to reveal occurrences on either of these two other types of grain boundaries. The low generation rates of $\mathrm{Ag}$ and low concentrations of $\mathrm{U}$, in combination with long diffusion distances, also may have prevented the concentrations of these elements from reaching critical levels needed to form a fission product precipitate. Additional areas and precipitates will need to be analyzed to resolve this observed behavior. However, the observations in this work would seem to justify a reconsideration of which $\Sigma$ values should actually be considered to possess "special" properties, at least for the precipitation of fission products. Furthermore, additional consideration of the grain boundary plane is needed as it can significantly affect the resulting grain boundary energy [33,35-37] and grain boundary diffusivity [30]. Thus, just a low CSL value may not be sufficient to inhibit precipitate formation and, ultimately, the grain boundary structure, as determined by both misorientation and the grain boundary plane, may be more relevant to fission product migration and precipitation behavior.

Table 4. Summary of CSL-related boundaries with precipitates.

\begin{tabular}{|c|c|c|c|c|c|c|}
\hline & \multicolumn{6}{|c|}{ Elements in Fission Product } \\
\hline & \multicolumn{2}{|c|}{ Pd Only } & \multicolumn{2}{|c|}{$\underline{\mathbf{P d}+\mathbf{A g}}$} & \multicolumn{2}{|c|}{$\underline{\mathbf{P d}+\mathbf{U}}$} \\
\hline & & $\begin{array}{c}\text { Number of } \\
\text { Grain }\end{array}$ & & $\begin{array}{c}\text { Number of } \\
\text { Grain }\end{array}$ & & $\begin{array}{c}\text { Number of } \\
\text { Grain }\end{array}$ \\
\hline & $\Sigma$ Value & Boundaries & $\Sigma$ Value & Boundaries & $\Sigma$ Value & Boundaries \\
\hline \multirow[t]{5}{*}{ Inner Sample } & 7 & 2 & $25 b$ & 1 & $19 a$ & 1 \\
\hline & $17 \mathrm{a}$ & 2 & & & $25 b$ & 1 \\
\hline & $19 a$ & 1 & & & & \\
\hline & $25 b$ & 1 & & & & \\
\hline & $29 b$ & 1 & & & & \\
\hline \multirow[t]{3}{*}{ Center } & 3 & 1 & - & - & - & - \\
\hline & 9 & 1 & & & & \\
\hline & $19 b$ & 1 & & & & \\
\hline Outer & - & - & - & - & - & - \\
\hline
\end{tabular}

Because comparison of the distributions of grain boundary precipitates is not statistically relevant between inner, center and outer samples in this study, it make sense to combine the data from the three samples and discuss the results as they pertain to the entire $\mathrm{SiC}$ layer. Specifically, fission product precipitates are most likely to occur on random, high-angle grain boundaries (Figure 9). As noted above, random, high-angle grain boundaries typically have higher grain boundary energies than low $\Sigma$, CSL-related grain boundaries, regardless of grain boundary plane [38]. It has been demonstrated that a linear relationship exists between grain boundary energy and grain boundary excess volume [32,33] and, thus, a larger excess volume is expected for random, high angle grain boundaries compared to either low-angle or low $\Sigma$, CSL-related grain boundaries. The fission product elements and $U$ have significantly larger atomic radii compared to either Si or C; strains caused by these elements would be minimized if located in grain boundaries with larger values of excess volume (e.g. random, high-angle grain boundaries). The diffusivity of a grain boundary is significantly affected by the excess grain boundary volume $[34,39,40]$. Thus, random, high-angle grain boundaries, with relatively large values of grain boundary energy (and, therefore, larger values of excess volumes) are considered to be the high diffusivity paths for impurity atoms [31]. The fraction of CSL-related grain boundaries containing fission products in Figure 9 is composed mostly of boundaries with a high $\Sigma$ value (see Table 4) that tend to exhibit larger grain boundary energy than boundaries associated with low values of $\Sigma$, e.g. $\Sigma 3$. Finally, diffusion of fission product elements in low angle grain boundaries is limited to dislocation pipe diffusion because these boundaries are composed of one or more arrays of discrete dislocations. 


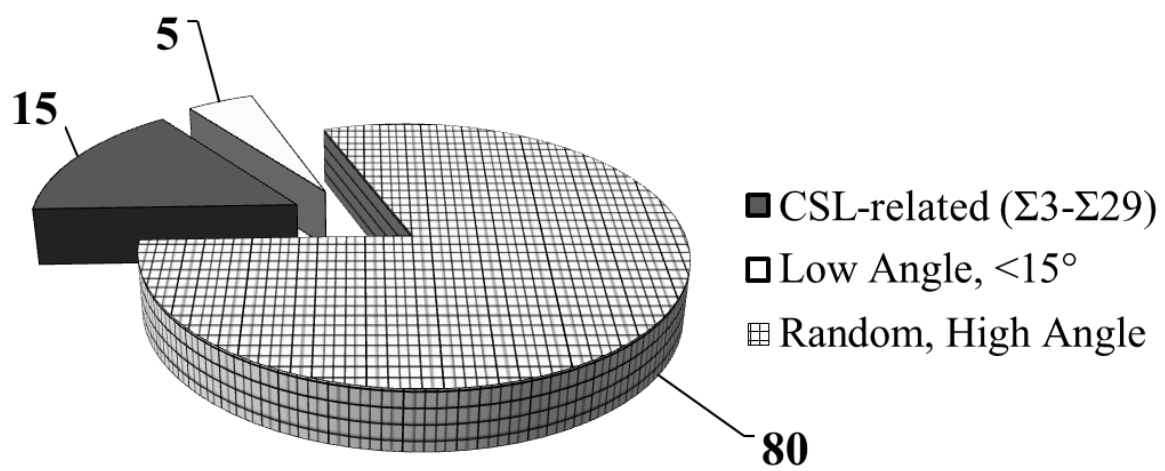

Figure 9. Distribution of the fission product precipitates on the various boundary types as a percentage.

\subsection{Relationship Between Specific Fission Product Elements and Grain Boundary Types}

Further analysis of the occurrence of specific fission product elements and combinations of them revealed some interesting trends related to grain boundary type that are summarized in Figure 10. Specifically, as mentioned above, precipitates that contain only Pd seem to occur on all types of grain boundaries (Figure 10a) with preference for random, high-angle grain boundaries. Again, Pd is found in precipitates at interior locations in $\mathrm{SiC}$ grains [7] and it is not surprising that precipitates with only Pd can be found on all three types of grain boundaries. However, when Pd was found in conjunction with either Ag or U, low-angle grain boundaries no longer appear to be viable defects for the formation of fission product precipitates (Figure 10c and d). The lack of precipitation on low-angle grain boundaries when either Ag or $\mathrm{U}$ are associated with Pd can be rationalized by noting the larger atomic radii of these two fission product elements, which could increase the strain energy of such precipitates on low-energy boundaries to the point where they are no longer energetically favorable. Finally, Ag was found to only occur on random, high-angle grain boundaries (Figure 10b) and never on low-angle or CSL-related grain boundaries. The presence of precipitates containing only Ag on only random, high-angle grain boundaries can be rationalized by both the larger atomic radius and the almost complete lack of solubility of $\mathrm{Ag}$ in $\mathrm{SiC}$ (i.e., a thermodynamic consideration). However, it is difficult to rationalize the presence of Ag only precipitates when Pd is present in the surroundings. Another study [41] also shows that Ag is capable of diffusing through $\mathrm{SiC}$, even when Pd is not present. However, if Pd were available, as is the case in the present study, then one would expect to find only $\mathrm{Pd}+\mathrm{Ag}$ precipitates due to the complete solubility of Ag in Pd [42] if the precipitates are metallic, solid solutions. If a particular boundary possesses a grain boundary energy and structure amenable for Ag only precipitates, which Fig. 10b implies that this will be a random, high angle grain boundary, then Pd would not be expected to be excluded from also being present on this boundary, based on its apparent ability to precipitate on grain boundaries of all energies. Figures 10a,c show $\mathrm{Pd}$ only and $\mathrm{Pd}+\mathrm{Ag}$ precipitates are prevalent on random, high angle grain boundaries. Thus, it is not clear what combination of grain boundary energy and structure could allow precipitation of Ag and yet prevent Pd from precipitating also. The possibility exists that Pd is actually present in the Ag-only precipitates found in this study but at levels below the limits of detection of the characterization methods. The same may be said of the Pd only precipitates - that Ag may actually be present but below the limits of detection. If so, then the grain boundary structure and/or energy of random, high angle grain boundaries somehow determines the ratio of Ag to Pd in the fission product precipitate, again implying that not all random, high angle grain boundaries should be considered equivalent or even similar when it comes to the segregation and transport of fission product elements. 
Conversely, if the precipitates are in the form of silicides then it might be possible to have Ag in solid solution in a Pd-silicide, to produce the $\mathrm{Pd}+\mathrm{Ag}$ precipitates, and yet not have $\mathrm{Pd}$ in solid solution in an Ag-silicide, to produce the Ag-only precipitates. However, Ag-silicides do not seem to occur [43]. Although, Olivier and Neethling [44] found that only very small amounts of Pd were necessary to assist Ag migration through SiC and Pd concentrations may be below the limits of detection used in this study. Therefore, the existence of Ag-only precipitates found in this study is unexplained at this time and will require determination of not only the crystal structure of the Ag-only precipitates but also that of the $\mathrm{Pd}$ and $\mathrm{Pd}+\mathrm{U}$ precipitates to fully understand the segregation of the fission product elements during (and after) irradiation. A more detailed understanding of the grain boundary energy and grain boundary structure also will be required.

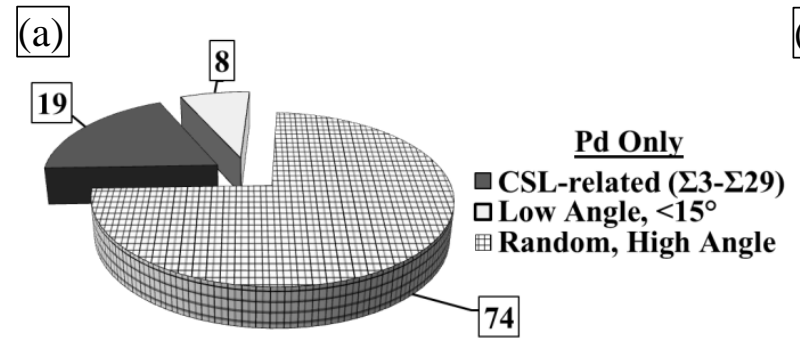

(b)
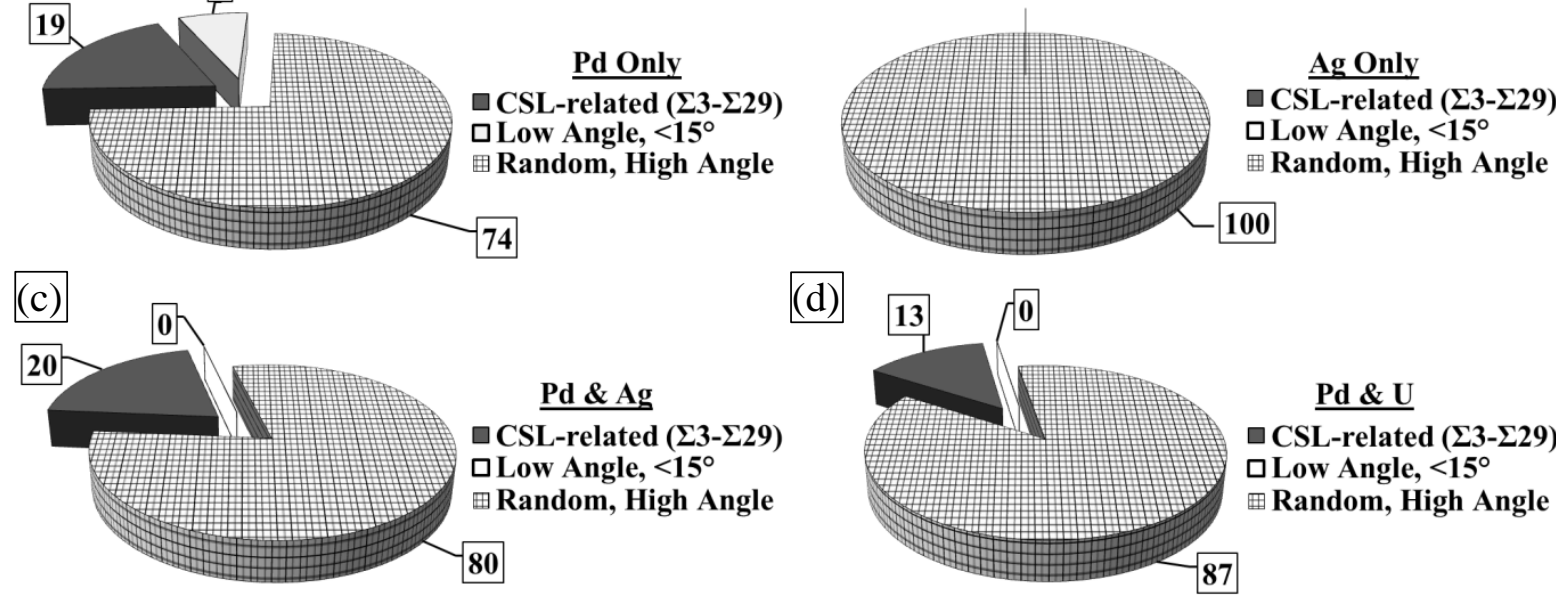

(d)

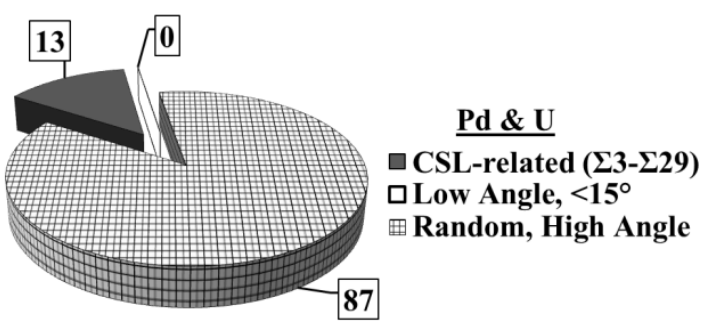

Figure 10. Distributions of fission products, as a percentage, by grain boundary type: (a) Pd only, (b) Ag only, (c) Pd+Ag, and (d) Pd+ U.

\section{CONCLUSIONS}

The objective of this study was to gather and correlate fission product composition data with the associated SiC grain boundary characteristics for the SiC layer of an irradiated TRISOcoated nuclear fuel particle. A secondary objective was to demonstrate application of a precession electron diffraction method for efficient characterization of the irradiated SiC layer grain boundary characteristics. The PED method developed earlier on unirradiated $\mathrm{SiC}$ was successfully used to determine the grain boundary misorientation angle and axis in the SiC layer of an irradiated TRISO fuel particle. It was also successfully demonstrated that detailed grain boundary characteristics in the vicinity of fission products could be established and compared to areas without fission products. Specific findings are listed here.

- The grain structure of the SiC layer in an irradiated TRISO fuel particle from the AGR-1 experiment is composed mainly of twin boundaries with a small fraction of low-angle grain boundaries (i.e., on the order of $10 \%$ ).

- The CSL-related grain boundary fraction increased from the IPyC/SiC interface to the $\mathrm{SiC} / \mathrm{OPyC}$ interface, mainly due to the increase of the twin boundary fraction, while the faction of random, high-angle grain boundaries decreased from the IPyC to the OPyC. 
- Fission product precipitates were commonly found on random, high-angle grain boundaries and to a lesser degree at low-angle and CSL-related grain boundaries.

- Pd was found at all types of grain boundaries, but most prominently on random, highangle grain boundaries.

- $\quad$ Pd-U and Pd-Ag precipitates were found on CSL-related and random, high-angle grain boundaries, but not on low-angle grain boundaries.

- Precipitates containing only Ag were only found on random, high-angle grain boundaries (i.e., not on either low-angle or CSL-related grain boundaries). The reason for Ag only precipitates is not known.

These results represent a preliminary study, which shows great promise for revealing the relationship between $\mathrm{SiC}$ grain boundary character and fission product migration through the SiC layer of TRISO-coated fuel particles. This study is recommended to be expanded to obtain information from more TRISO particles with the same fabrication and irradiation history to enable final conclusions to be drawn from a more representative dataset.

Additionally, it is recommended that efforts be made to analyze the grain boundary plane, in addition to the other grain boundary parameters, to fully understand the influence of grain boundaries on fission product migration through the SiC layer of TRISO and ultimately, fission product release during irradiation.

\section{REFERENCES}

1 Demkowicz, P. A., J. D. Hunn, R. N. Morris, J. M. Harp, P. L. Winston, C. A. Baldwin, and F. C. Montgomery, 2012, Paper HTR2012-3-021, Proceedings of the HTR 2012, Tokyo, Japan, October 28 - November 1, 2012.

2 Nabielek, H. and P. E. Brown, 1975, "The Release of Silver-110m in High Temperature Reactors: Technical Note,” OECD Dragon Project 657: 370.

3 Nabielek, H., P. E. Brown, and P. Offermann, 1977, Nuc. Tech. 35: 483-493.

4 Minato, K., T. Ogawa, S. Kashimura, K. Fukuda, M. Shimizu, Y. Tayama, and I. Takahashi, 1990, J. Nuc. Matls. 172: 184-196.

5 Engelhard, J., K. Krüger, H. Gottaut, 1975, "Investigations of the Impurities and Fission Products in the AVR Coolant at an Average Hot Temperature of $950^{\circ} \mathrm{C}$,” Nucl. Eng. Design 34: 85.

6 van Rooyen, I. J., D. E. Janney, B. D. Miller, P. A. Demkowicz, and J. Riesterer, 2014, Nucl. Eng. Design 271: 114-122.

7 Lillo, T. M. and I. J. van Rooyen, 2015, “Associations of Pd, U and Ag in the SiC Layer of Neutron-Irradiated TRISO Fuel,” J. Nuc. Matls 460: 97-106.

8 van Rooyen, I. J., T. M. Lillo, and Y. Q. Wu, 2014, J. Nucl. Mater. 446: 178-186

9 Schrader, D., S. M. Khalil, T. Gerzcak, T. R. Allen, A. J. Heim, I. Szlufarska, D. Morgan, 2011, J. Nucl. Mater. 408: 257-271.

10 Khalil, S., N. Swaminathan, D. Schrader, A. J. Heim, D. D. Morgan, I. Szlufarska, 2011, Phys. Rev. B 84: 214104.

11 Friedland, E., J.B. Malherbe, N.G. van der Berg , T. Hlatshwayo, A.J. Botha, E. Wendler, W. Wesch, 2009, J. Nuc. Mater. 389:326-331. 
12 Xiao, H.Y., Y. Zhang, L.L. Snead, V. Shutthanandan, H.Z. Xue, W.J. Weber, 2012, J. Nuc. Mater. 420:123-130.

13 Gerczak, T.J., B. Leng, K. Sridharan, J.L. Hunter Jr, A.j. Giordani, T.R. Allen, 2015, J. Nuc. Mater. 461: 314-324.

14 J.B. Malherbe, 2013, J. Phys.D: Appl. Phys. 46: 473001.

15 Maclean, H. J., 2004, "Silver Transport in CVD Silicon Carbide,” Ph.D. thesis, Department of Nuclear Engineering, MIT, Boston.

16 van Rooyen, I. J., M. L. Dunzik-Gougar, and P. M. van Rooyen, 2014, Nucl. Eng. Design 271: 180-188.

17 Szlufarska, I., D. D. Morgan, S. Khalil, D. Shrader, and A. Heim, 2010, ANS Trans. 102:751-752.

18 Khalil, S., N. Swaminathan, D. Shrader, A. Heim, D. D. Morgan, and I. Szlufarska, 2011, Phy. Rev. B 84: 214104.

19 Rabone, J. and E. López-Honorato, 2014, J. Nuc. Matls. doi: http//dx.doi.org/10.1016/j.jnucmat.2014.11.032.

20 Lillo, T., I. J. van Rooyen, and Y. Wu, 2015, Nuc. Eng. Design, submitted September 2015, to be published.

21 Hunn, J. D. and R. A Lowden, 2006, “Data Compilation for AGR-1 Baseline Coated Particle Composite LEU01-46T,” ORNL/TM-2006/019, Revision 1, April 2006.

22 ASTM Standard E2627-13, Standard Practice for Determining Average Grain Size Using Electron Backscatter Diffraction (EBSD) in Fully Recrystallized Polycrystalline Materials.

23 O’Connell, J. H. and J. H. Neethling, 2014, J. Nucl. Mater. 445: 20-25.

24 Tan, L., T. R. Allen, J. D. Hunn, and J. H. Miller, 2008, J. Nucl. Mater. 372: 400-404.

25 Long, Y., A. Javed, Z. Chen, X. Xiong, and P. Xiao, 2013, Int. J. Appl. Ceram. Technol. 10: 11-19.

26 Kirchhofer, R., Hunn, J.D., Demkowicz, P.A., Cole, J.I. and Gorman, B.P., 2013, J. Nuc. Matls., vol. 432, 127-134.

27 Sterbentz, J. W. and H. D. Gougar, 2011, “Comparison of Fission Product Buildup in Candidate NGNP Fuel and the AGR-1 TRISO-Particle Irradiation Test,” Technical Evaluation Study, TEV-1301, Idaho National Laboratory.

28 Hunn, J. D., R. N. Morris, C. A. Baldwin, F. C. Montgomery, C. M. Silva, and T. J. Gerczak, 2013, “AGR-1 Irradiated Compact 4-4-2 PIE Report: Evaluation of AsIrradiated Fuel Performance with Leach Burn Leach, IMGA, Materialography, and Xray Tomography,” ORNL/TM-2013/236, 90 pages.

29 Kleykamp, H. and Kang, S.G., 1996, “Pd-U Phase Diagram,” ASM Alloy Phase Diagrams Center, P. Villars, editor-in-chief and H. Okamoto and K. Cenzual, section editors, http://www1.asminternational.org/AsmEnterprise/APD, ASM International, Materials Park, $\mathrm{OH}$.

30 Priester, L., 1989, Rev. Phys. Appl., 24: 419-438.

31 Peterson, N.L., 1983, Int. Met. Rev., 28: 65-91.

32 Wolf, D., 1989, Scripta Met., 23:1913-1918.

33 Wolf, D., 1990, Acta metall. mater., 38: 791-798.

34 Randle, V., 1997, Acta mater., 46: 1459-1480. 
35 Lojkowski, W., H. Gleiter and R. Maurer, 1988, Acta metall., 36:69-79.

36 Wolf, D., 1990, Acta metall. mater., 38:781-790.

37 Herrmann, G, H. Gleiter and G. Baro, 1976, Acta metall., 24: 353-359.

38 Goodhew, P.J., 1979, Met. Sci, 13: 108-112.

39 Randle, V., 2004, Acta mater., 52: 4067-4081.

40 Shewmon, P.G., Transformations in Metals, 1969, McGraw-Hill Book Co., New York, p. 63.

41 Lopez-Honorato, E., H. Zhang, D. Yang and P. Xiao, 2011, J. Am. Ceram. Soc., 94: 3064-3071.

42 Morioka, S. and Hasebe, M., 1990, “Ag-Pd Phase Diagram,” ASM Alloy Phase Diagrams Center, P. Villars, editor-in-chief and H. Okamoto and K. Cenzual, section editors, http://www1.asminternational.org/AsmEnterprise/APD, ASM International, Materials Park, $\mathrm{OH}$.

43 Hassam, S., Ågren, J., Gaune Escard, M., Bros, J.P., 1990, “Ag-Si Phase Diagram,” ASM Alloy Phase Diagrams Center, P. Villars, editor-in-chief and H. Okamoto and K. Cenzual, section editors, http://www1.asminternational.org/AsmEnterprise/APD, ASM International, Materials Park, $\mathrm{OH}$.

44 Olivier, E. J. and J. H. Neethling, 2013, J. Nuc. Mater. 432: 252-260.

\section{ACKNOWLEDGEMENTS}

This work was sponsored by the U.S. Department of Energy, Office of Nuclear Energy, under U.S. Department of Energy Idaho Operations Office Contract DE-AC07-05ID14517, as part of the Very High-Temperature Reactor Development Program and as part of an Advanced Test Reactor Nuclear Science User Facilities Experiment. The United States Government retains and the publisher, by accepting the article for publication, acknowledges that the United States Government retains a nonexclusive, paid-up, irrevocable, world-wide license to publish or reproduce the published form of this manuscript, or allow others to do so, for United States Government purposes. Mary Lou Dunzik-Gougar is thanked for her review of this paper. 